\title{
MAIN CHALLENGES OF STATE POLICY OF HIGHER EDUCATION IN GEORGIA
}

\section{GIULI GIGUASHVILI}

Academic Doctor of Economics, Professor

Gori State Teaching University, Georgia

giuligiguashvili@gmail.com

\section{TAMAR MAKASARASHVILI}

Academic Doctor of Economics, Professor

Gori State Teaching University, Georgia

tmakasarashvili@gmail.com

Abstract. The proper education policy of the state and the efficiency of investment invested in the education system will ensure the success of the education system. Reforms in Georgia are going on as a continuous process. Despite some positive results, the state failed to provide sustainable development of the system under poor financing.

The research aims to study the problems related to the quality of education, its financing and availability. Evaluation of the main directions of the state policy of education and elaboration of certain recommendations for the improvement of the system.

In the paper opinions are expressed on higher education budgeting, promotion of education financing as part of social responsibility of business, main challenges of the new government initiative - „Education - Road to Freedom” are discussed.

KEYWORDS: EDUCATION POLICY, HIGHER EDUCATION FINANCING.

For citation: Giguashvili, G., Makasarashvili, T. (2020). Main Challenges of State Policy of Higher Education in Georgia. Globalization and Business, №9, pp. 163-170 (In Georgian). https://doi.org/10.35945/gb.2020.09.020 


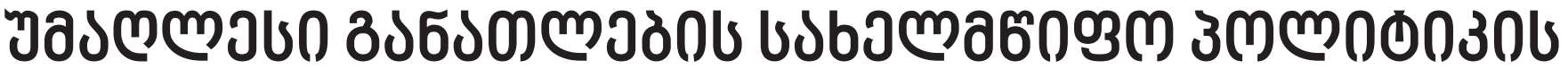

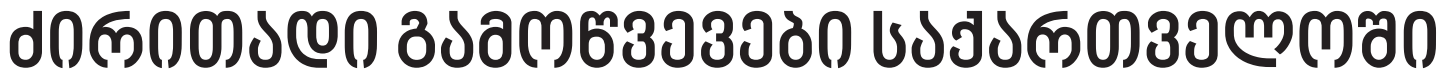

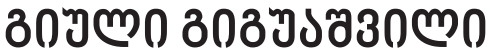

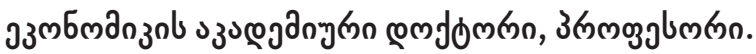

zмmol buby

giuligiguashvili@gmail.com

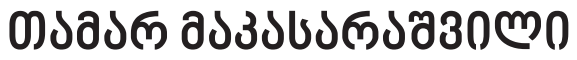

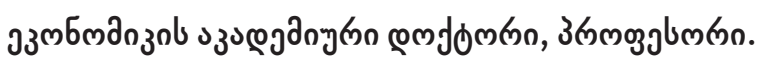

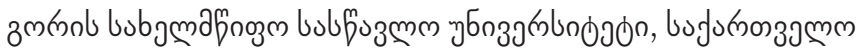

tmakasarashvili@gmail.com

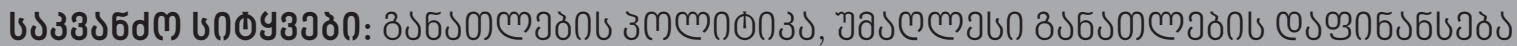

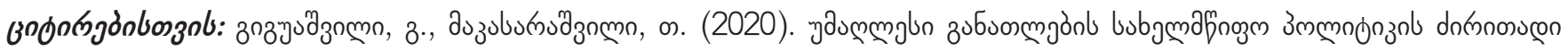

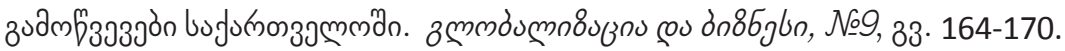

https://doi.org/10.35945/gb.2020.09.020

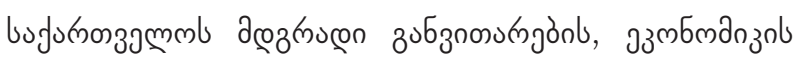

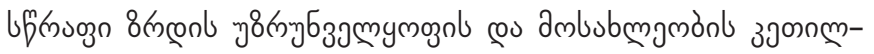

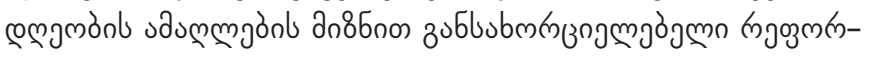

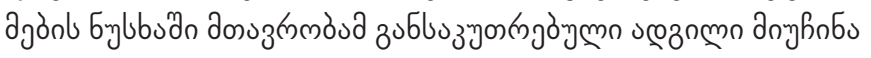

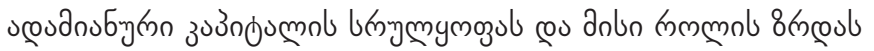

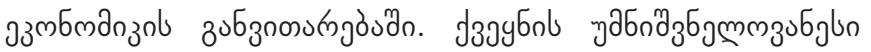

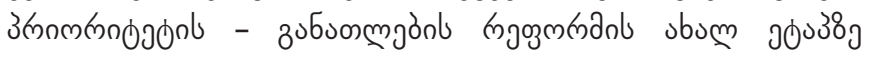

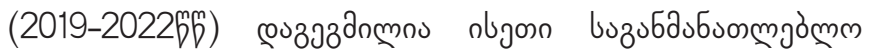

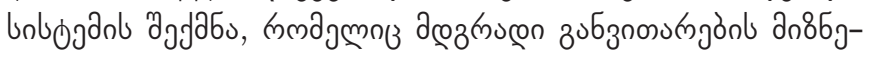

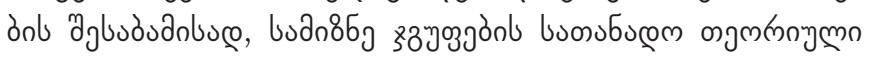

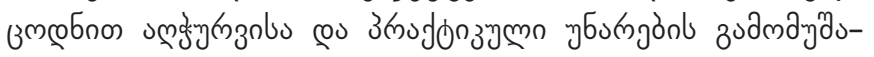

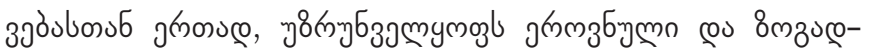

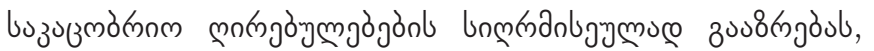

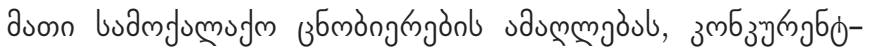

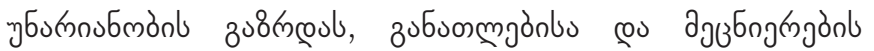

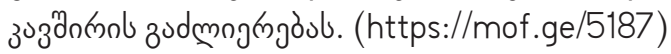

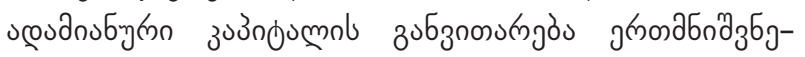

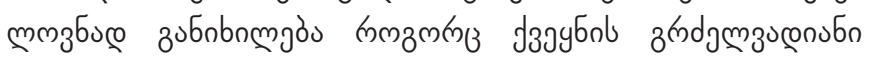

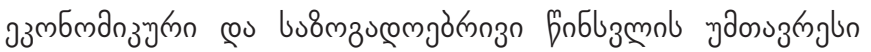

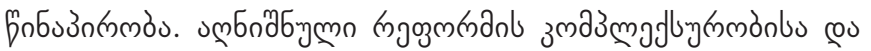

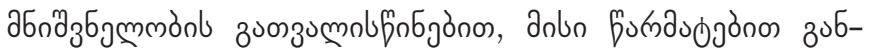

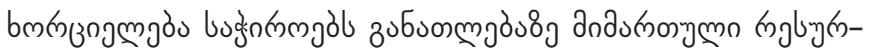
ugonb 8m

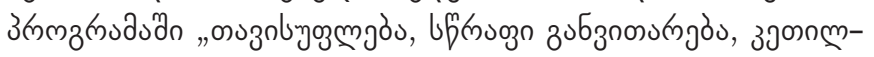

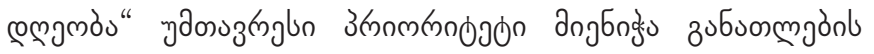

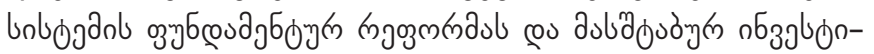

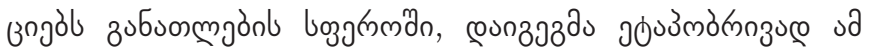

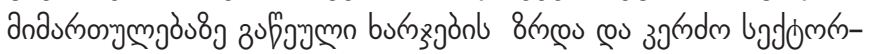

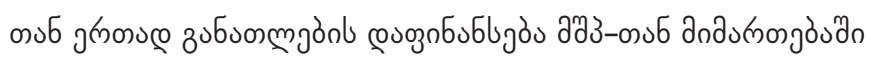

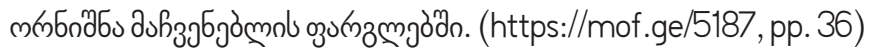

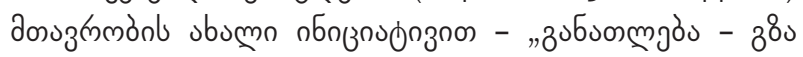

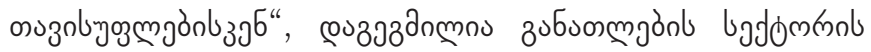

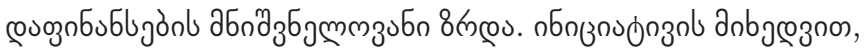

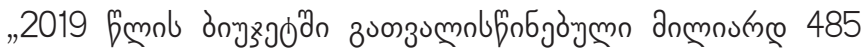

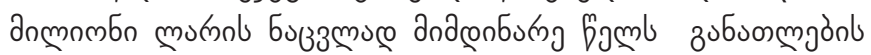

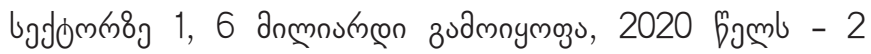

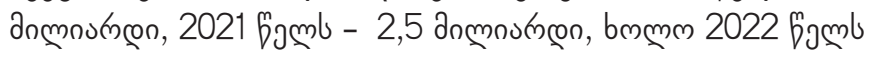

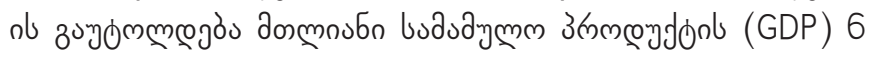

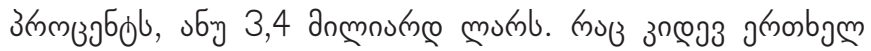

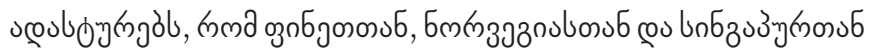

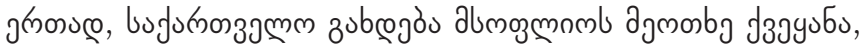

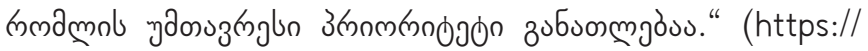
commersant.ge/)

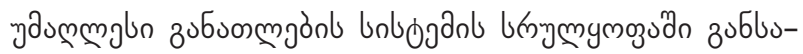

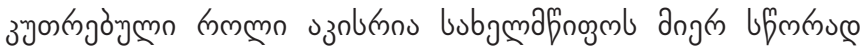

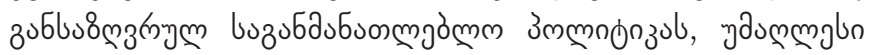

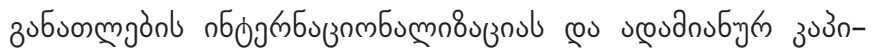

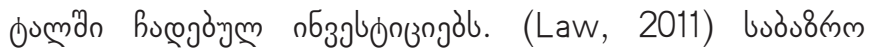

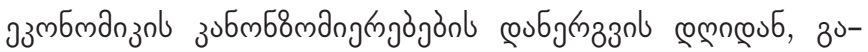

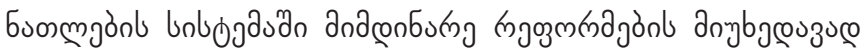

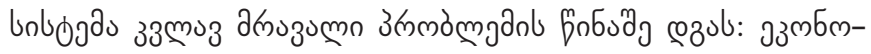

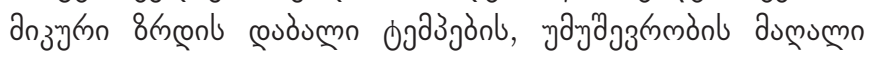

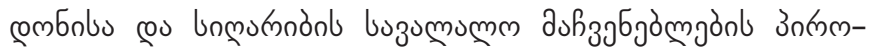

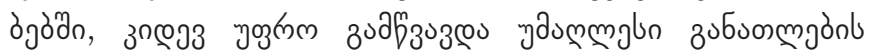

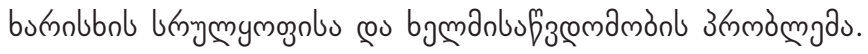

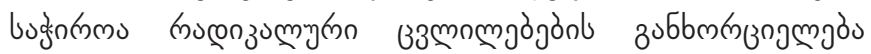




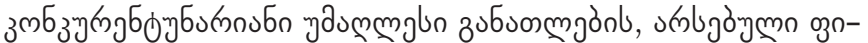

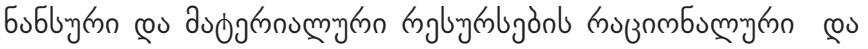

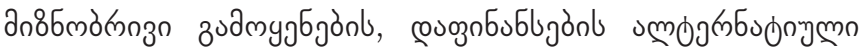

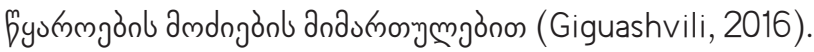

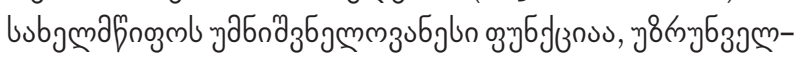
ymb bufurnoszamml zmblbond

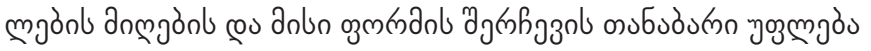

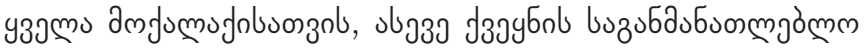

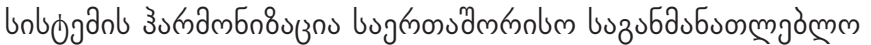

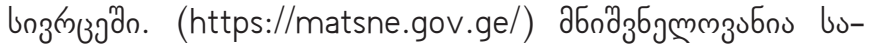

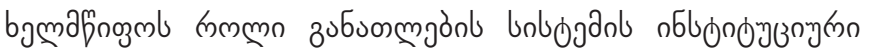

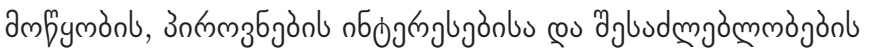

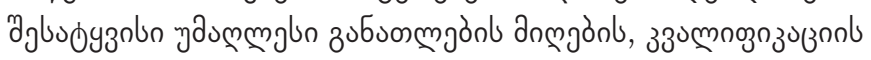

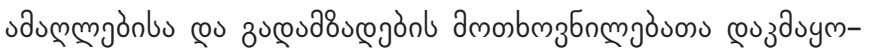

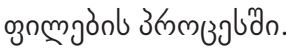

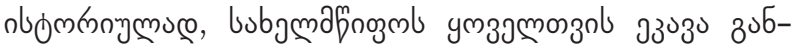

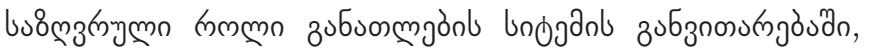

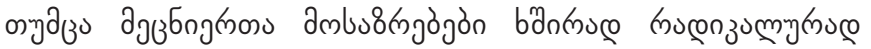

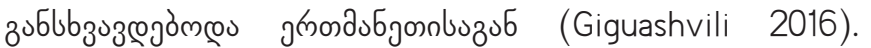

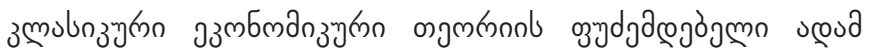

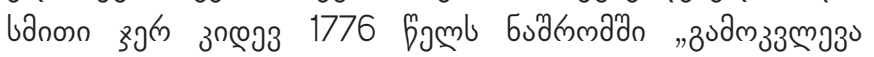

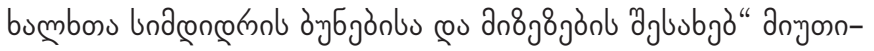

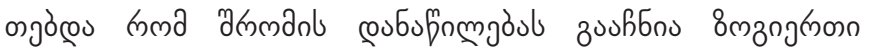

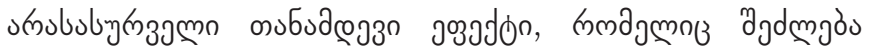

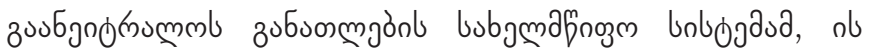

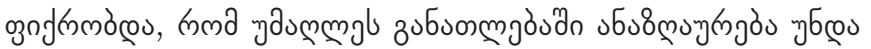

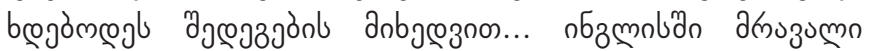

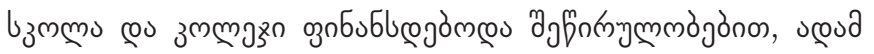

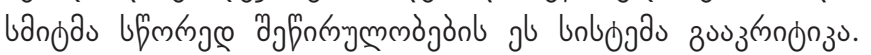

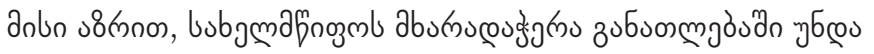

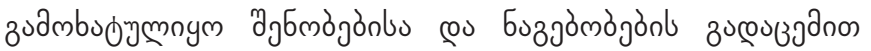

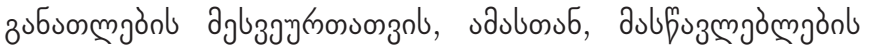

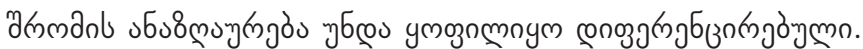
(Sartania, 2001:96)

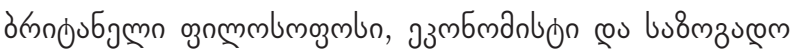

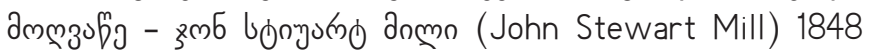

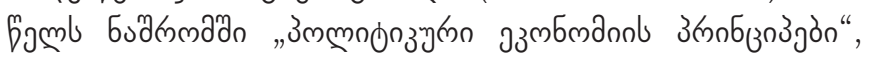

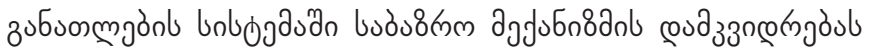

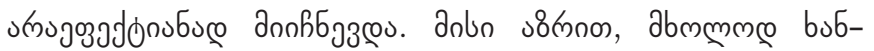

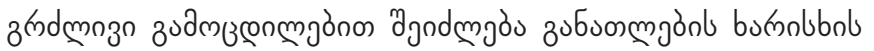

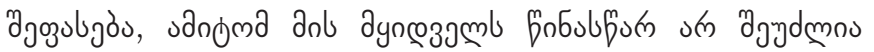

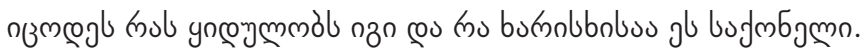

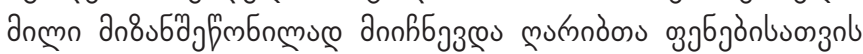

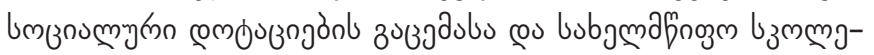

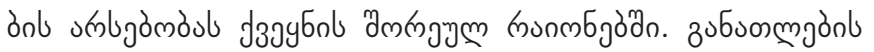

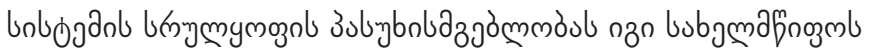

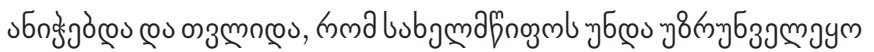

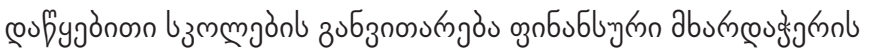
88̊no. (Sartania, 2001:97)

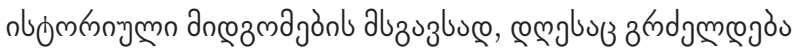

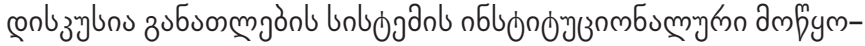

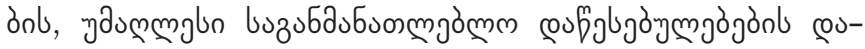

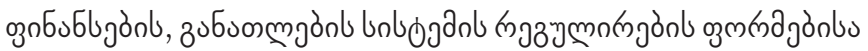

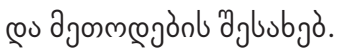

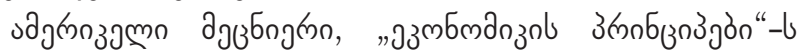

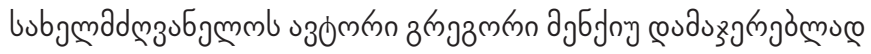

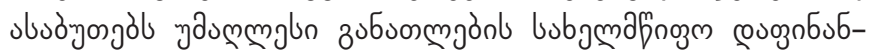

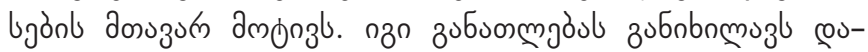

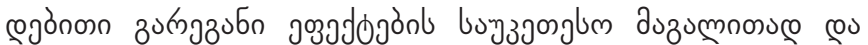

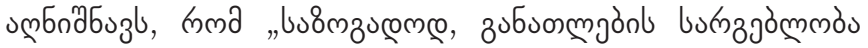

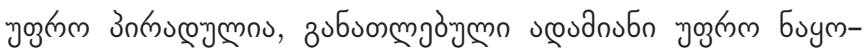

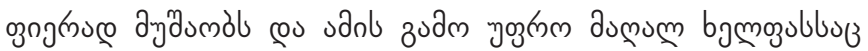

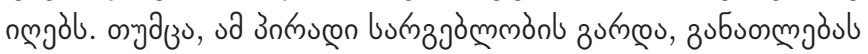

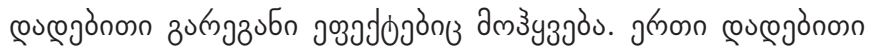

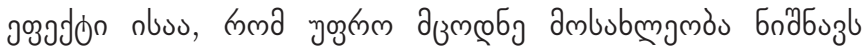

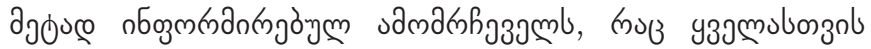
चzgorglo anuz

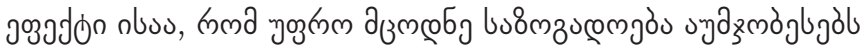

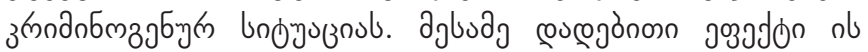

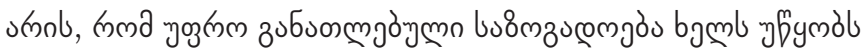

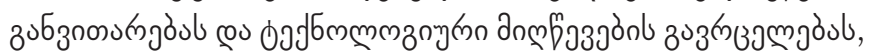

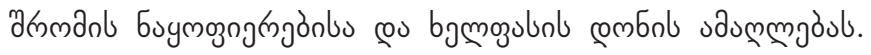

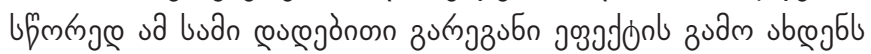

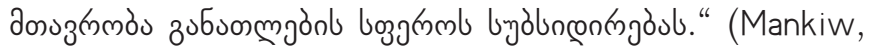
2008:225)

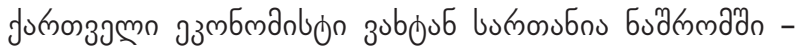

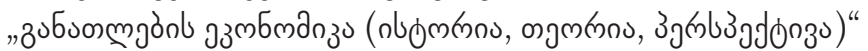

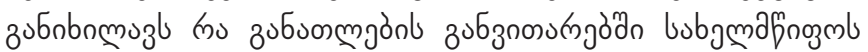

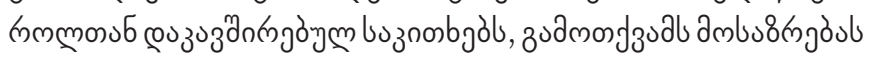

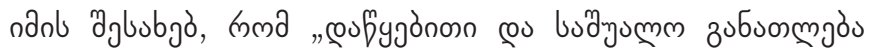

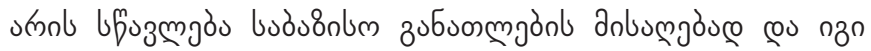

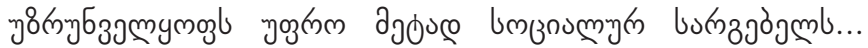

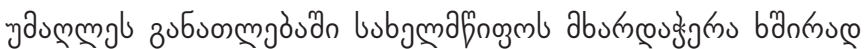

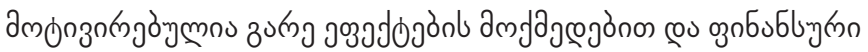

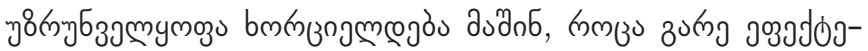

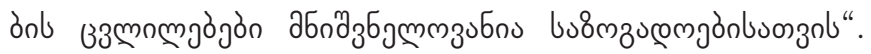

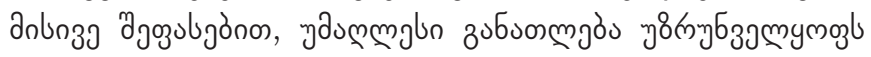

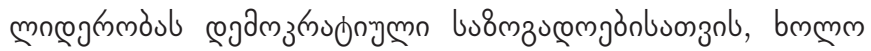

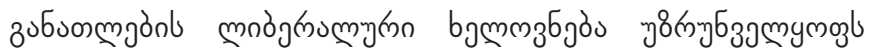

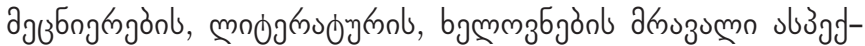

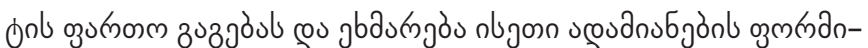

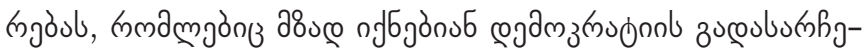

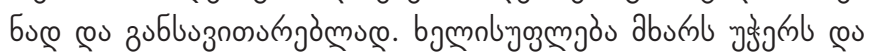

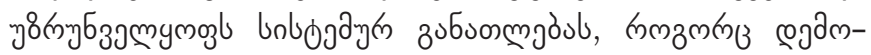
z

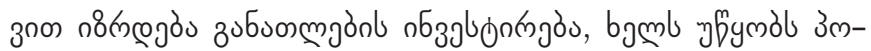

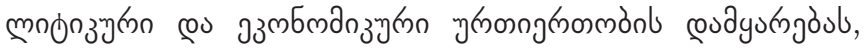

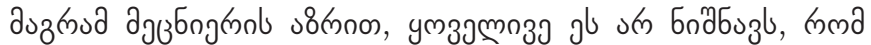

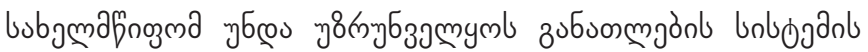
y3ams Enyublon. (Sartania, 2001:103) 


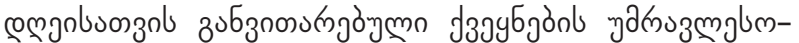

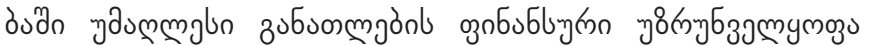

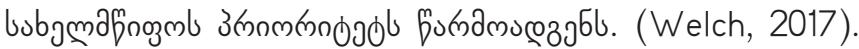

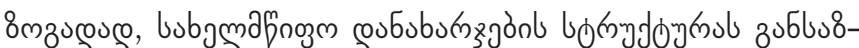

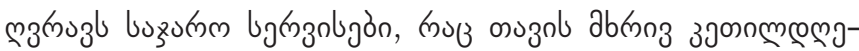

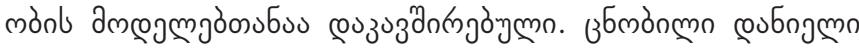

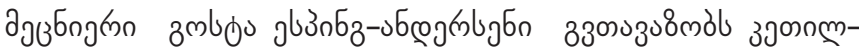

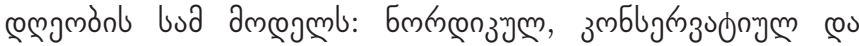

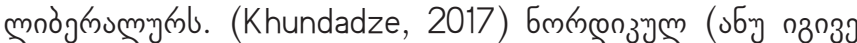

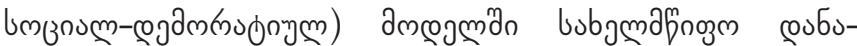

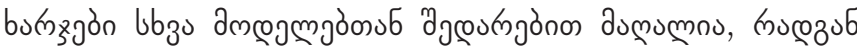

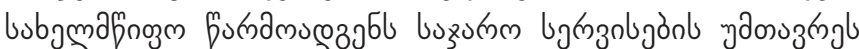

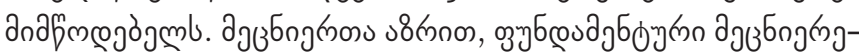

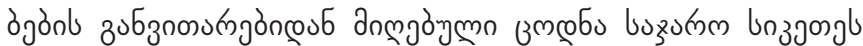

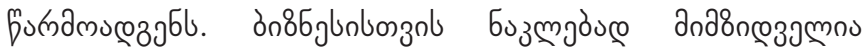

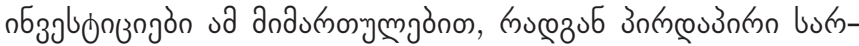
зjommo

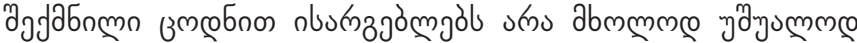

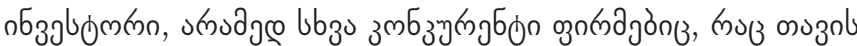

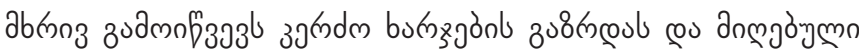

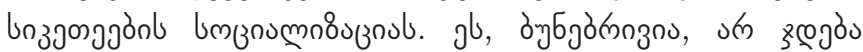

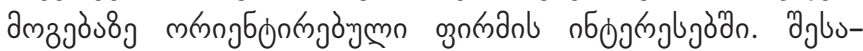

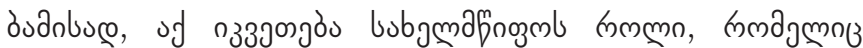

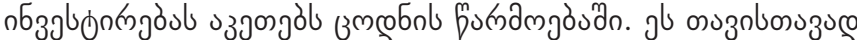

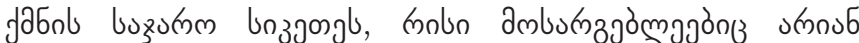

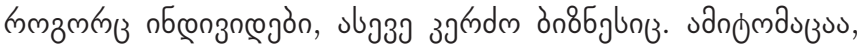

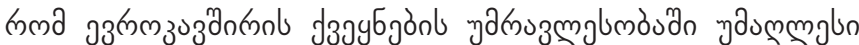

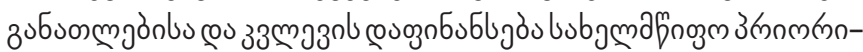

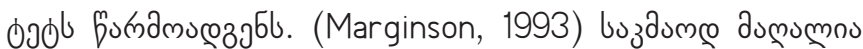

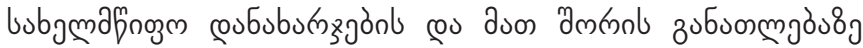

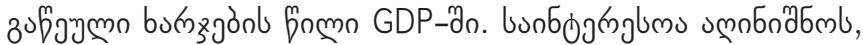

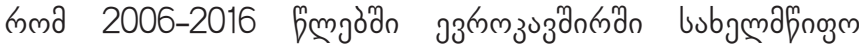

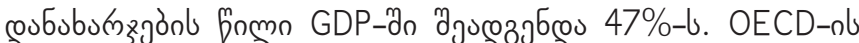

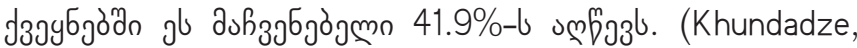

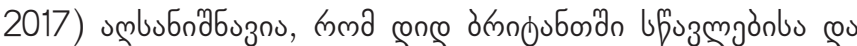

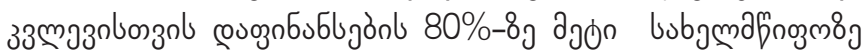
amenl (Barbara, 2011).

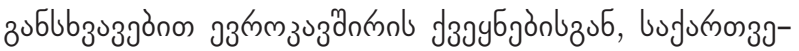

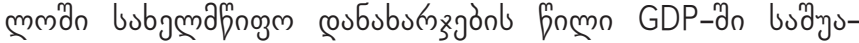

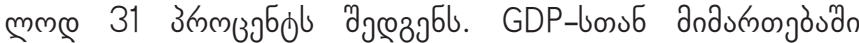

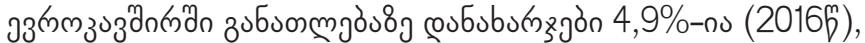

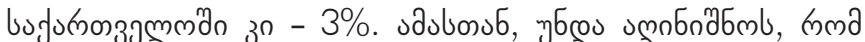

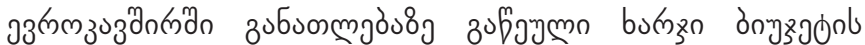

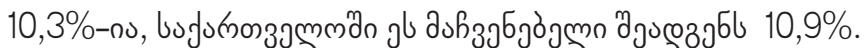

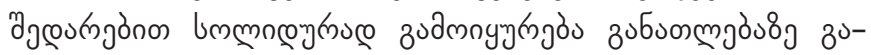

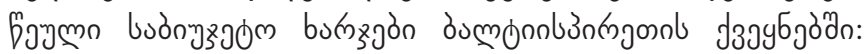

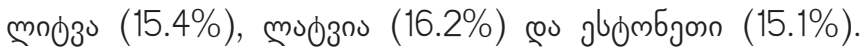
(Khundadze, 2017)

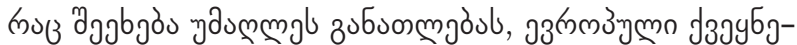

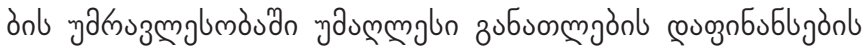

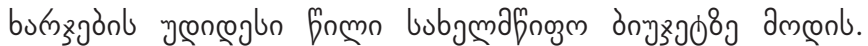

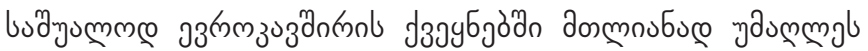

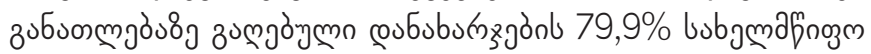

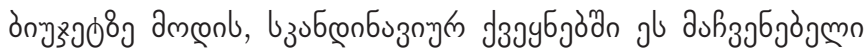

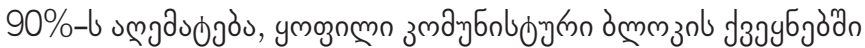

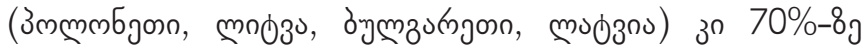

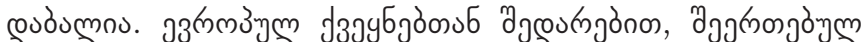

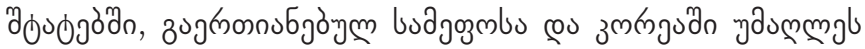

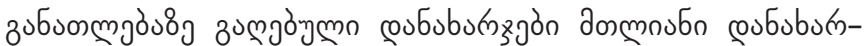

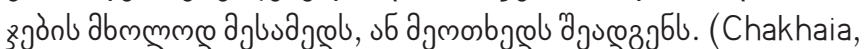
2013)

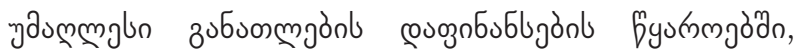

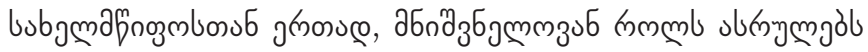

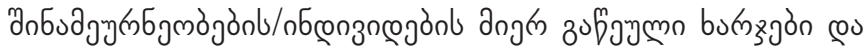

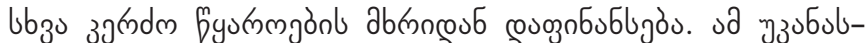

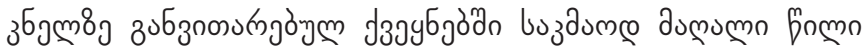

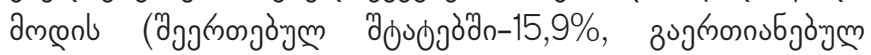

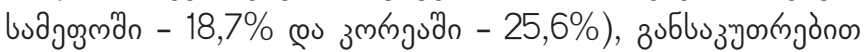

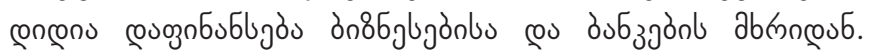

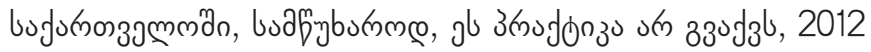

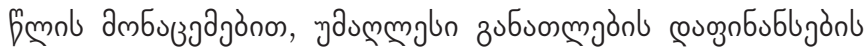

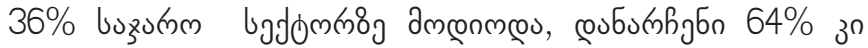

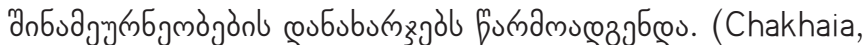

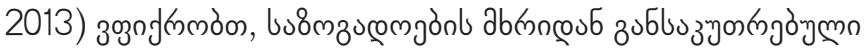

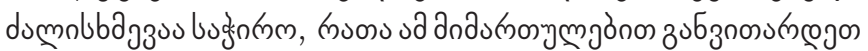

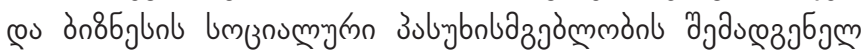

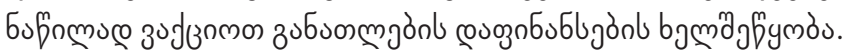

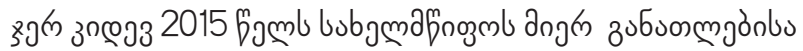

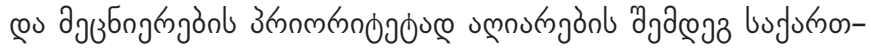

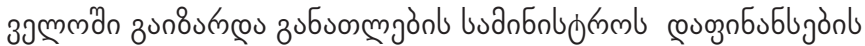

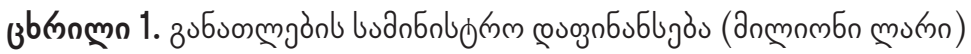

\begin{tabular}{|c|c|c|c|c|c|c|c|c|}
\hline & $2012 f$. & 20136. & $2014 f$. & 20156. & 20166. & 2017 f. & 20180 . & 20196. \\
\hline 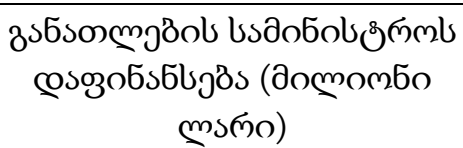 & 626,8 & 675,9 & 741,1 & 840,8 & 975,2 & 1172.5 & 1236.6 & $1,485,0$ \\
\hline 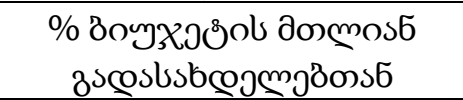 & 8,0 & 8,3 & 8,2 & 8,7 & 9,6 & 10,9 & 12,9 & 11,3 \\
\hline
\end{tabular}

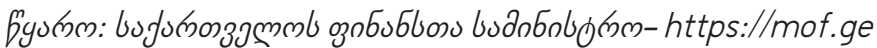




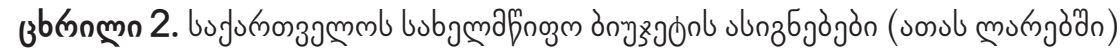

\begin{tabular}{|c|c|c|c|c|c|c|c|}
\hline \multirow[b]{2}{*}{ змलूо } & \multirow[b]{2}{*}{ coslusbjomjas } & \multirow{2}{*}{ 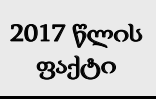 } & \multirow{2}{*}{$\begin{array}{l}2018 \text { fొmol } \\
303 \text { as }\end{array}$} & \multicolumn{4}{|c|}{2019 6mols 3082s } \\
\hline & & & & 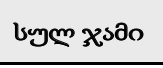 & 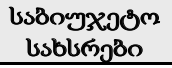 & 3గురకిం & 3ణృழంఠిం \\
\hline 0000 & bym zsan & $11,764,835.4$ & $12,459,500.0$ & $13,090,000.0$ & $11,696,250.0$ & $92,700.0$ & $1,301,050.0$ \\
\hline 3200 & 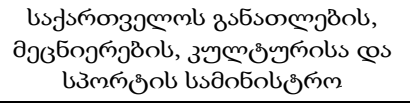 & $1,397,210.7$ & $1,461,270.0$ & $1,508,500.0$ & $1,469,600.0$ & $38,900.0$ & 0.0 \\
\hline 3204 & 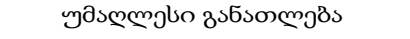 & $135,865.4$ & $148,505.0$ & $168,960.0$ & $168,960.0$ & 0.0 & 0.0 \\
\hline $\begin{array}{c}3204 \\
03\end{array}$ & 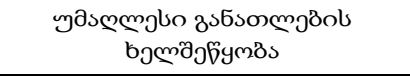 & 151.3 & 150.0 & 170.0 & 170.0 & 0.0 & 0.0 \\
\hline $\begin{array}{c}3204 \\
04\end{array}$ & 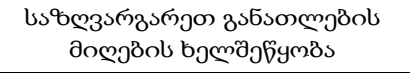 & $6,771.4$ & $6,910.0$ & $6,910.0$ & $6,910.0$ & 0.0 & 0.0 \\
\hline $\begin{array}{c}3204 \\
05\end{array}$ & 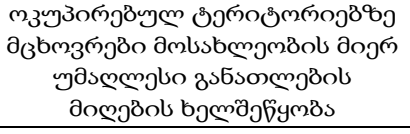 & 0.0 & 0.0 & 200.0 & 200.0 & 0.0 & 0.0 \\
\hline $\begin{array}{c}3204 \\
06\end{array}$ & 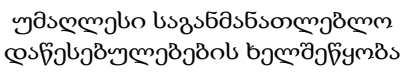 & $24,246.1$ & $16,015.0$ & $14,560.0$ & $14,560.0$ & 0.0 & 0.0 \\
\hline 3205 & 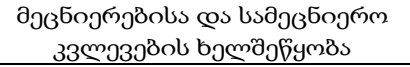 & $61,421.6$ & $65,600.0$ & $65,470.0$ & $65,470.0$ & 0.0 & 0.0 \\
\hline
\end{tabular}

fyurnm: https://matsne.gov.ge/

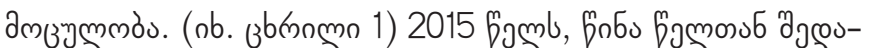

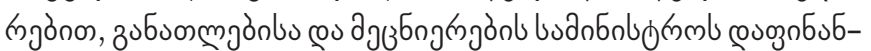

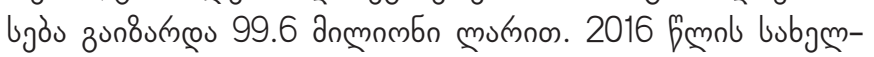

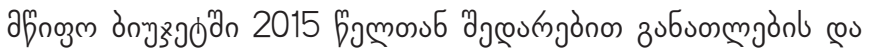

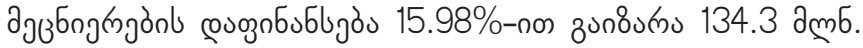

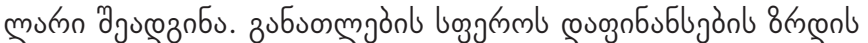

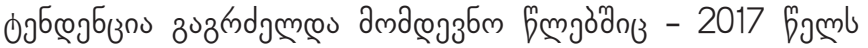

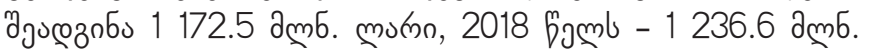
mukn, 2019 fmenl on

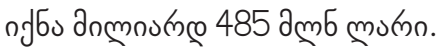

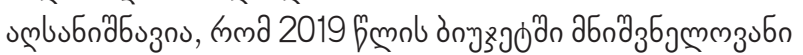

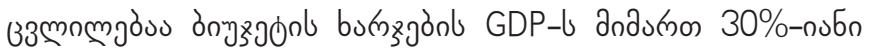

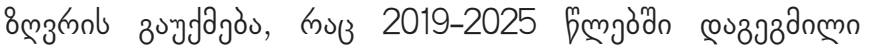

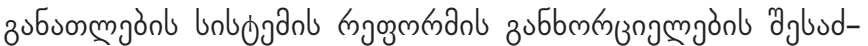

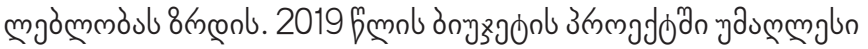

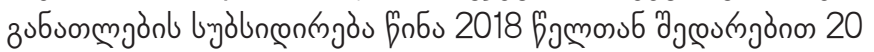

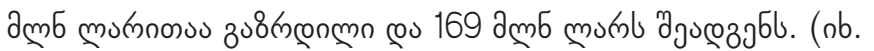

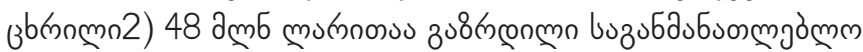

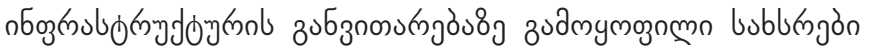

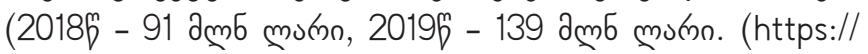
www.transparency.ge/)

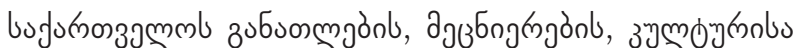

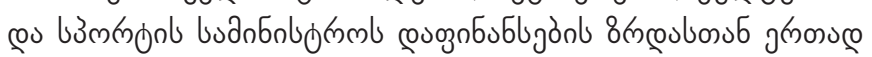

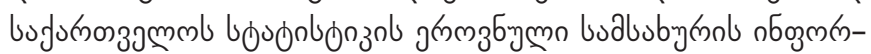

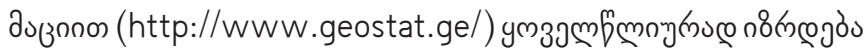

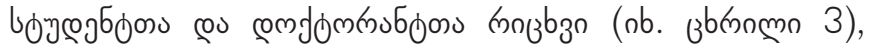

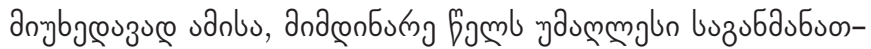

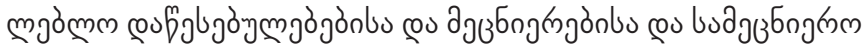

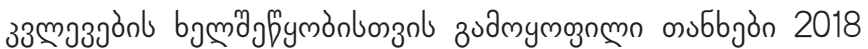

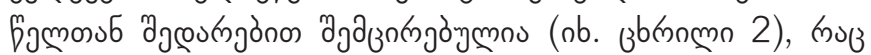

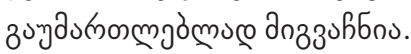

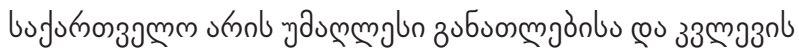

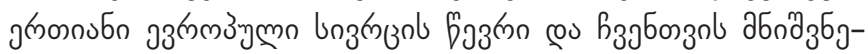

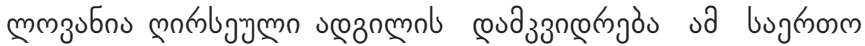

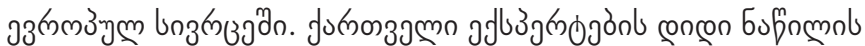

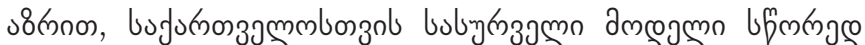

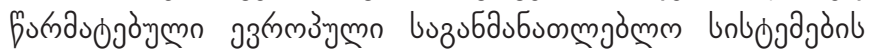

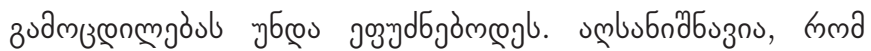
चб

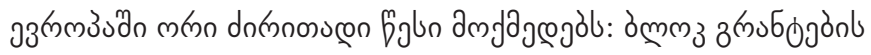

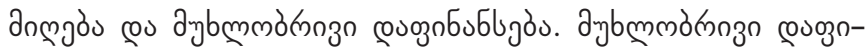

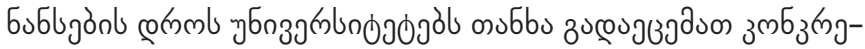

вbronmo 3.

\begin{tabular}{|c|c|c|c|c|c|c|c|}
\hline & 2012 & 2013 & 2014 & 2015 & 2016 & 2017 & 2018 \\
\hline 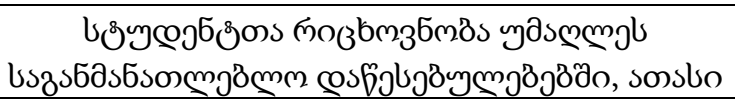 & 109.5 & 117.7 & 124.2 & 132.9 & 140.3 & 143.8 & 147.7 \\
\hline 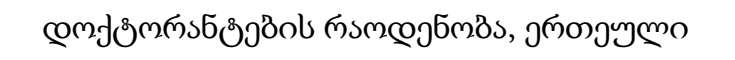 & 3040 & 3213 & 3410 & 3765 & 4076 & 3977 & 3512 \\
\hline
\end{tabular}

fyurnm: http://www.geostat.ge/?action=page\&p_id=205\&lang=geo 


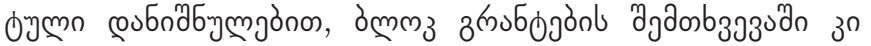

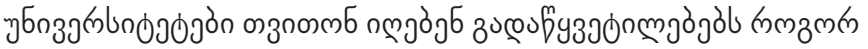

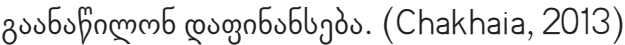

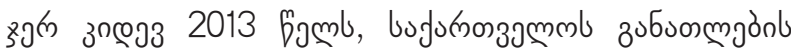

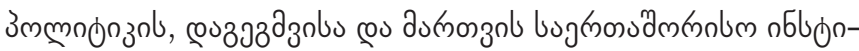
oyddo (EPPM - The International Institute for Education)

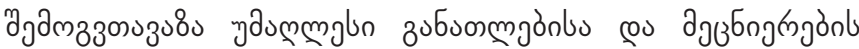

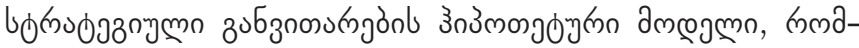

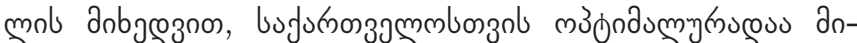

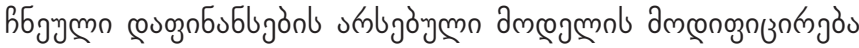

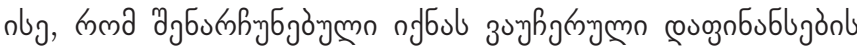

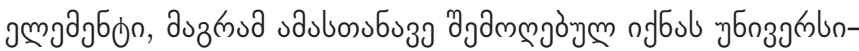

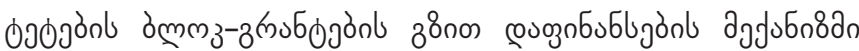

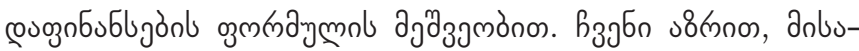

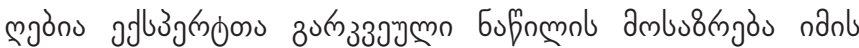

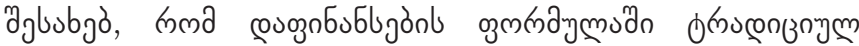

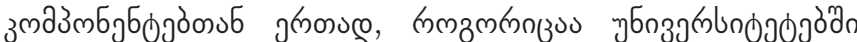

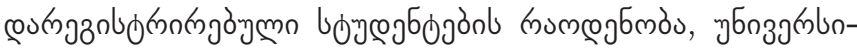

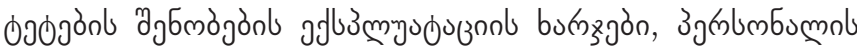

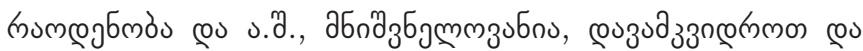

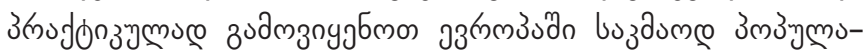

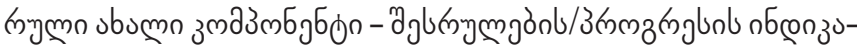

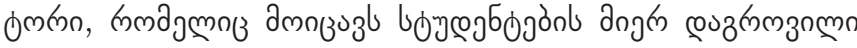

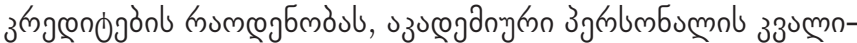

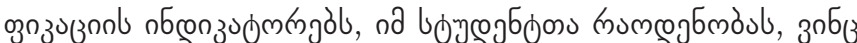

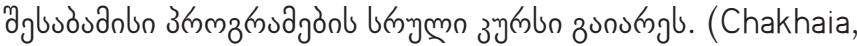
2013)

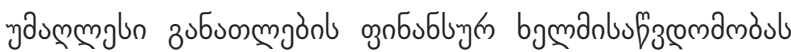

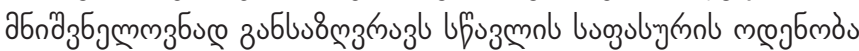

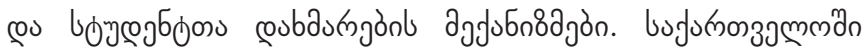

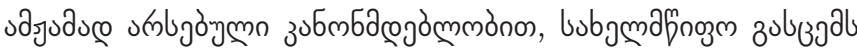

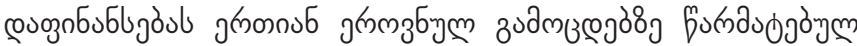

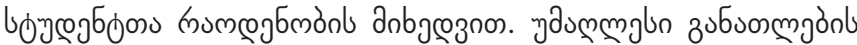

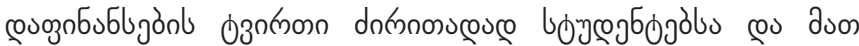

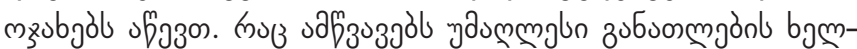

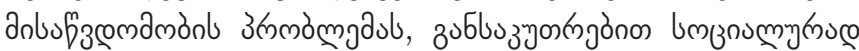

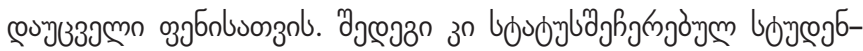

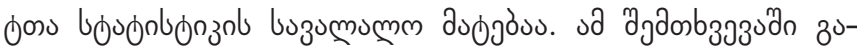

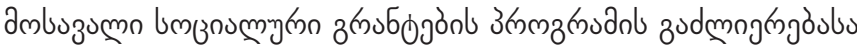

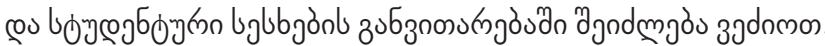

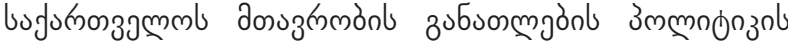

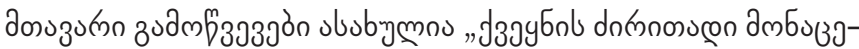

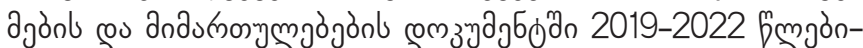

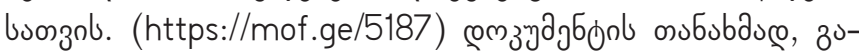

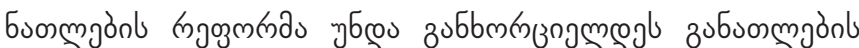

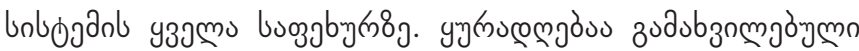

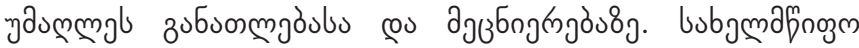

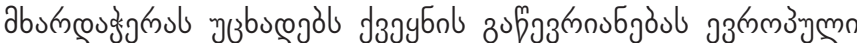

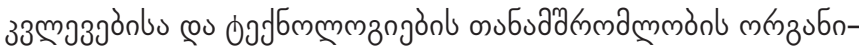

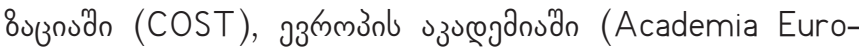

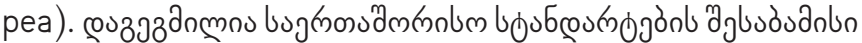

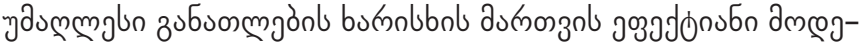

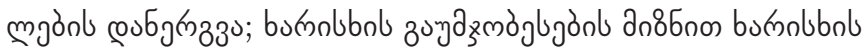

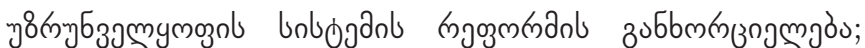

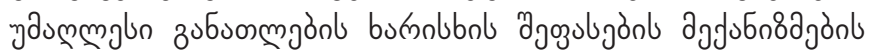

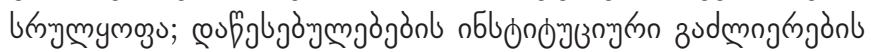

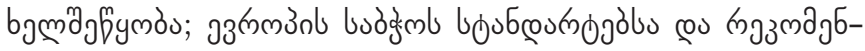

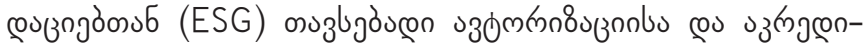

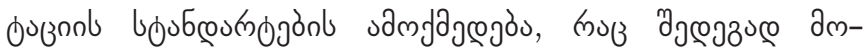

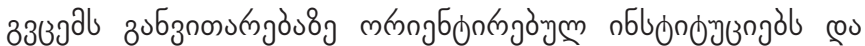

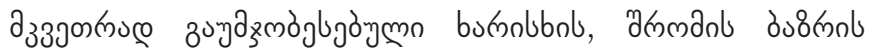

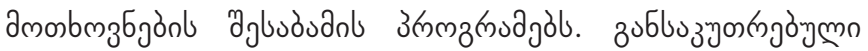

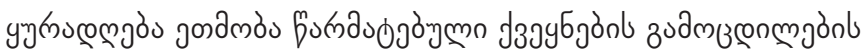

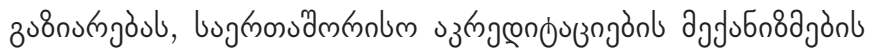

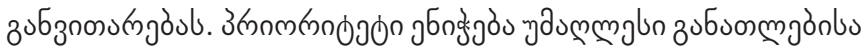

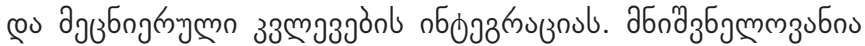

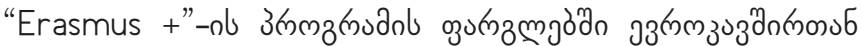

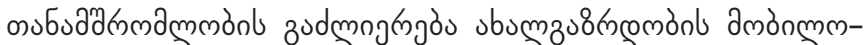

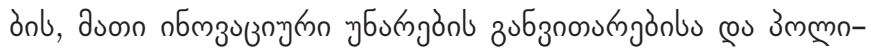

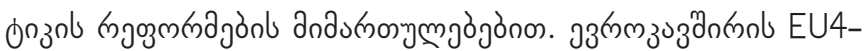

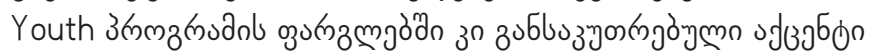

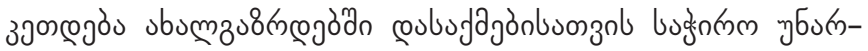

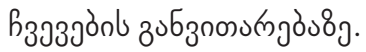

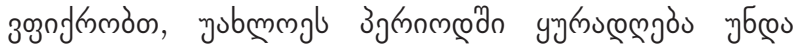

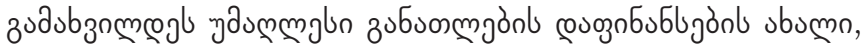

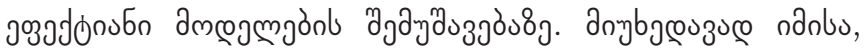

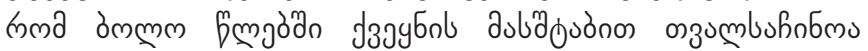
चб audgrnoumyn-

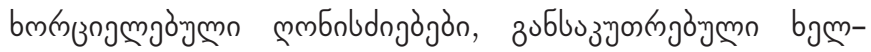

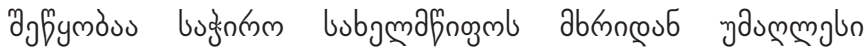

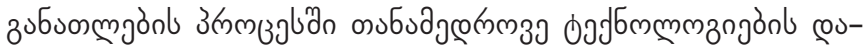

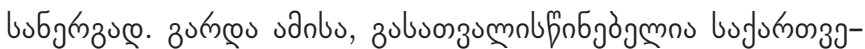

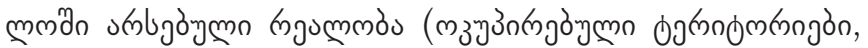

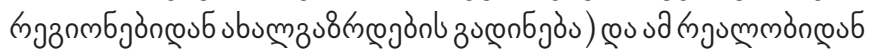

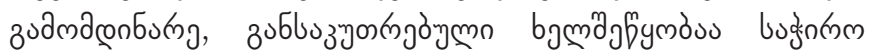

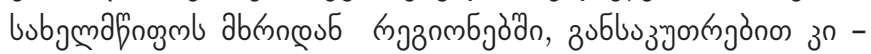
bu8

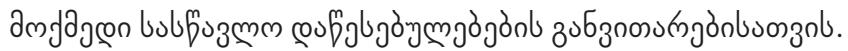

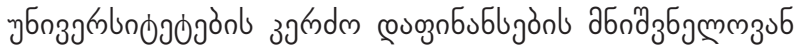

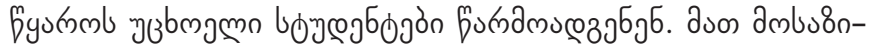

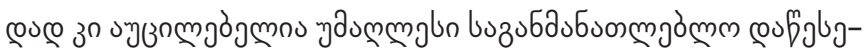

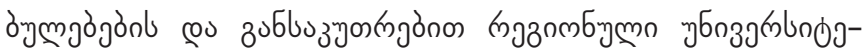

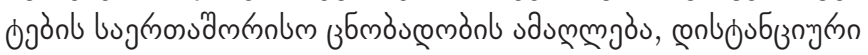

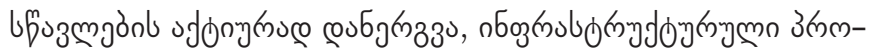

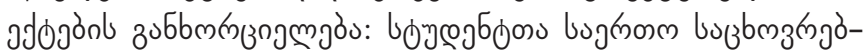

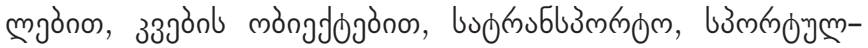

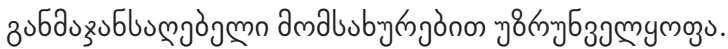

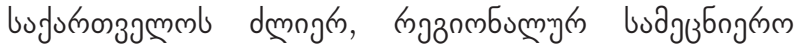

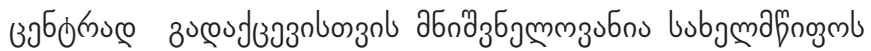




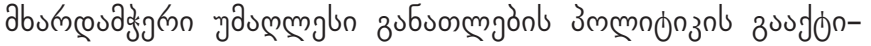

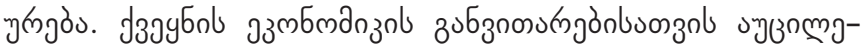

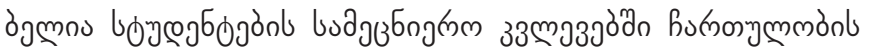

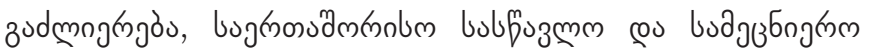

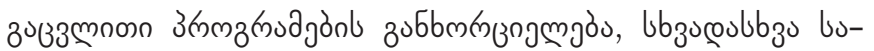

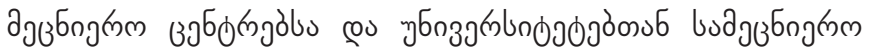

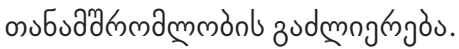

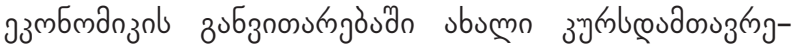

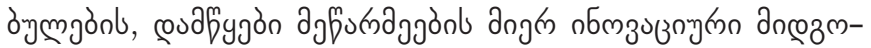

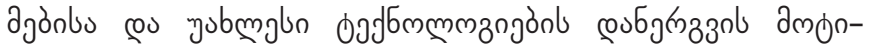

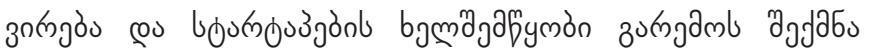

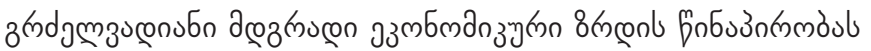

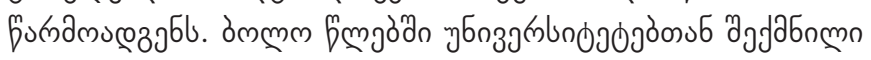

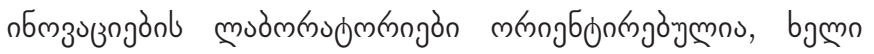

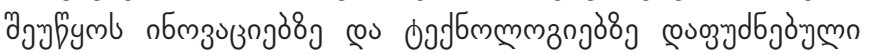

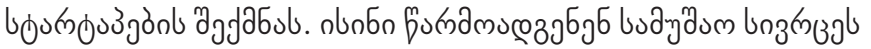

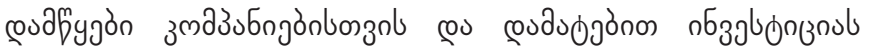

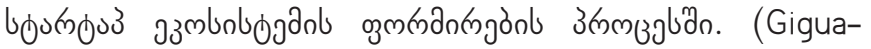
shvili,.2018) bufunnosgmmm

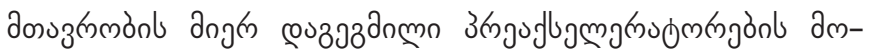

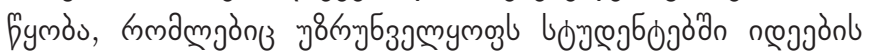

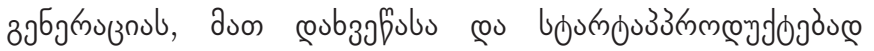

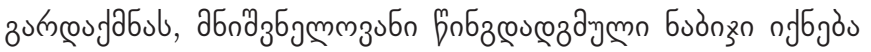

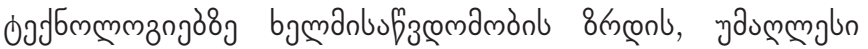

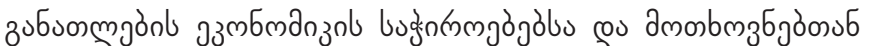

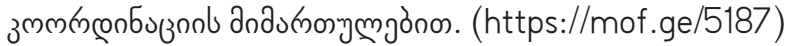

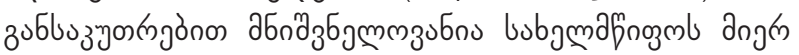

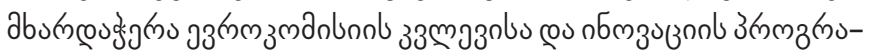

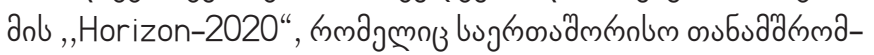

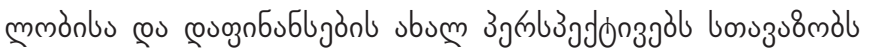

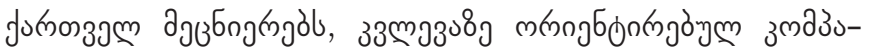

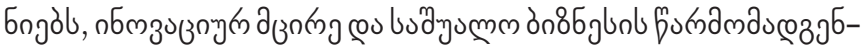

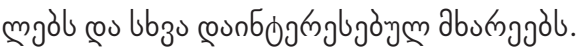

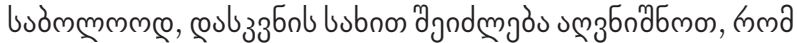

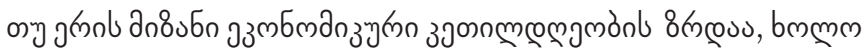

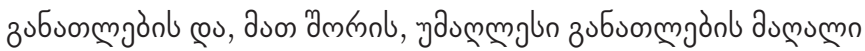

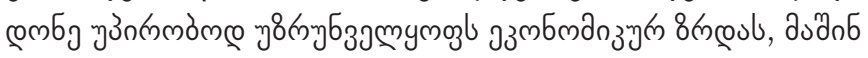

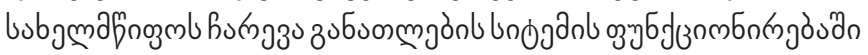

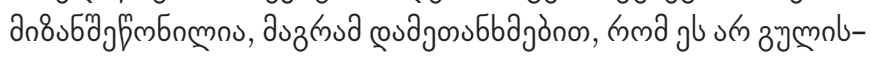

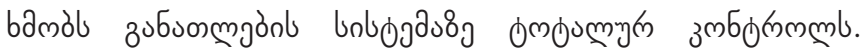

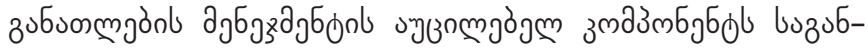

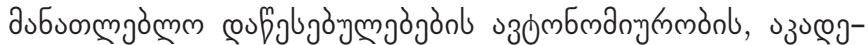

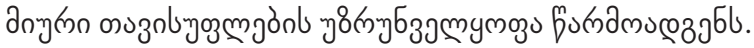

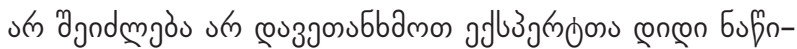

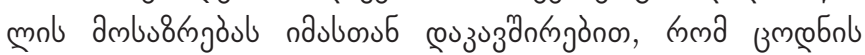

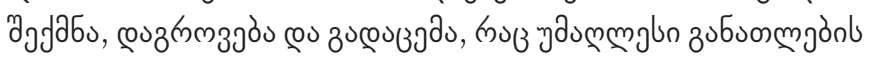

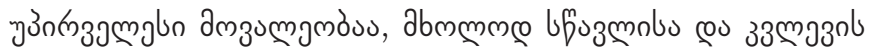

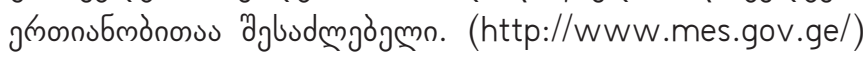

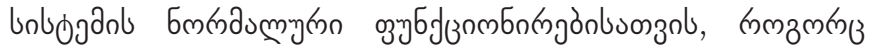

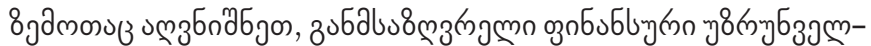

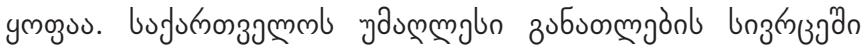

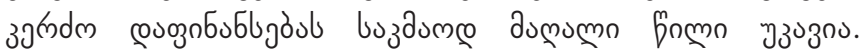

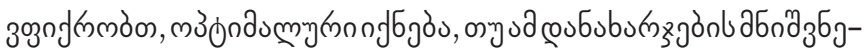

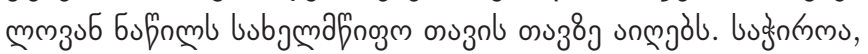

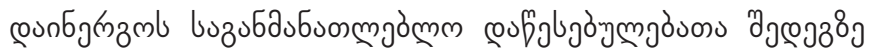

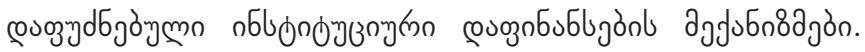

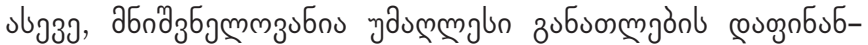

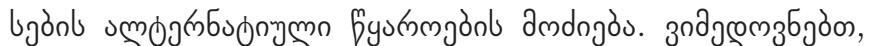

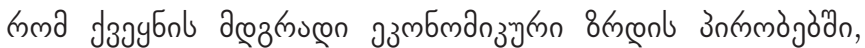

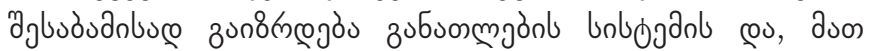

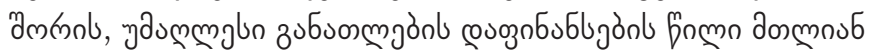

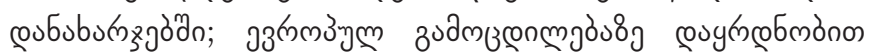

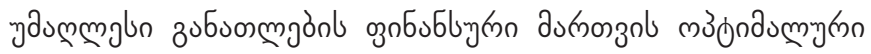

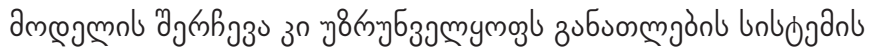

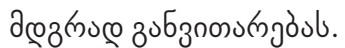

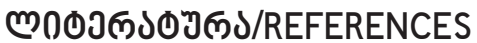

Giguashvili G. (2016). Financial Aspects of the Management of Higher Education in Georgia. International Scientific Conference - «Education Management: Current Challenges and Development Perspectives» November 18-19, 2016. Gori State Teaching University. 2017. pp. 172. (In Georgian)

Giguashvili G., Makasarashvili T. (2018). Peculiarities of Innovation Infrastructure Formation in Georgia, The Eleventh International Scientific Conference "Education and Science in the XXI Century: Reality, Challenges, Perspectives», Gori State Teaching University, November 16-17, 2018, Gori, Georgia. (In Georgian)

N. Gregory Mankiw (2008), Principles of Economics

Sartania V. (2001). Economics of Education (History, Theory, Perspective); Tb. 2001(In Georgian)

Chakhaia L. (2013). Higher Education and Research Funding and Financial Management. URL: https://www.tsu.ge/data/file_ $\mathrm{db} /$ xarisxis_martvis_dep/ganatlebis-politika-2.pdf. (In Georgian)

Khundadze T. (2017). Government Expenditures in Georgia and the European Union, URL: https://1tv.ge/analytics/ sakhelmwifo-danakharjebi-saqartvelosa-da-evrokavshirshi/ -16.08.2017 (In Georgian) https://mof.ge/5187: Ministry of Finance of Georgia, Document of Basic Data and Directions of the Country for 2019-2022. (In Georgian) https:// commersant.ge/: URL: https://commersant.ge/ge/post/premieri-2019-wlis-biudjetshi-ganatlebis-seqtorze-1-6-miliardi- 
lari-iqneba-gatvaliswinebuli-2022-wels-ki-mshp-s-6-s-gautoldeba, 2009-03-08 (In Georgian) https://matsne.gov.ge/: The Legislative Herald of Georgia, Constitution of Georgia, https://matsne.gov.ge/ka/document/view/30346 (In Georgian) https://www.transparency.ge/ - 2019 Draft State Budget of Georgia: Analysis and Recommendations, URL: https:// www.transparency.ge/ge/blog/sakartvelos-2019-clis-saxelmcipo-biujetis-proektis-analizi-da-rekomendaciebi,October 18, 2018 (In Georgian) https://matsne.gov.ge/ - The Legislative Herald of Georgia, Law of Georgia, The State Budget of Georgia for 2019. (In Georgian) URL: https://matsne.gov.ge/ka/document/view/4419670?publication=0 http://www. geostat.ge/ - National Statistics Office of Georgia, URL: http://www.geostat.ge/?action=page\&p_id=205\&lang=geo (In Georgian) http://www.mes.gov.ge/ - Ministry of Education, Science, Culture and Sport of Georgia, Strategic Directions of the Education and Science System Development, URL: http://www.mes.gov.ge/uploads/strategia..pdf (In Georgian)

Law, J., \& Van-Cauter, K. (2011). Internationalising Higher Education: The Role of the British Council. In P. Lane \& M. Fraser (Eds.), Franco-British Academic Partnerships: The Next Chapter (pp. 166-176). Liverpool University Press. doi:10.5949/ UPO9781846316777.024

Welch, A. (2017). Higher Education and the Developmental State. In T. Carroll \& D. Jarvis (Eds.), Asia after the Developmental State: Disembedding Autonomy (Cambridge Studies in Comparative Public Policy, pp. 359-387). Cambridge: Cambridge University Press. doi:10.1017/9781316480502.015

Marginson, S. (1993). Economic policies in higher education. In Education and Public Policy in Australia (pp. 122-142). Cambridge: Cambridge University Press. doi:10.1017/CBO9780511559389.007

Barbara J. Gabrys, Jane A. Langdale (2011). The higher education system. Cambridge University Press. Book: How to Succeed as a Scientist. 2011, pp 202-207. DOI:https://doi.org/10.1017/CBO9781139015561 